\title{
Neutrino Masses, Dark Matter and B-L Symmetry at the LHC
}

\author{
Tong $\mathrm{Li}^{1,2 *}$ and Wei $\mathrm{Chao}^{2 \dagger}$ \\ ${ }^{1}$ Bartol Research Institute, Department of Physics and Astronomy, \\ University of Delaware, Newark, DE 19716, USA \\ ${ }^{2}$ Center for High Energy Physics, Peking University, Beijing 100871, P.R. China
}

(Dated: August 29, 2018)

\begin{abstract}
We establish a hybrid seesaw mechanism to explain small neutrino masses and predict cold dark matter candidate in the context of the $B-L$ gauge symmetry extension of the Standard Model. In this model a new scalar doublet and two new fermion singlets are introduced at loop-level beyond the minimal Type I seesaw. The lightest particle inside the loop can be dark matter candidate. We study in detail the constraints from neutrino oscillation data, lepton flavor violating processes and cosmological observation. We also explore the predictions of the decays of the new charged scalars in each spectrum of neutrino masses and show the most optimistic scenarios to distinguish the spectra. We consider the pair production of the stable fermion associated with two observable SM charged leptons at the LHC, which occurs in a two-step cascade decay of the new gauge boson $Z^{\prime}$ and the new charged scalars stand as intermediate particles. The masses of missing dark matter and its parent particle can be well-determined in such production topology.
\end{abstract}

\section{INTRODUCTION}

The observation of neutrino oscillations [1 4 4] has revealed that neutrinos have small but nonzero masses, which cannot be accommodated in the Standard Model (SM) without introducing extra components. Besides, the SM does not provide any candidate for the non-baryonic cold dark matter (DM), which has been confirmed by precisely cosmological observations [5]

$$
\Omega_{D} h^{2}=0.1123 \pm 0.0035
$$

There are also some possible DM events from direct search setting upper-limit on the DM-nucleon scattering cross-section [6-10], although they still cannot be interpreted as significant evidence. To accommodate these observations, the minimal SM must be extended. As so, the dark matter and neutrino physics offer an ideal window to search for physics beyond SM.

\footnotetext{
* Electronic address: nklitong@hotmail.com

† Electronic address: chaow@pku.edu.cn
} 
It is well known that neutrinos can be Dirac or Majorana fermions. Up to now, the most attractive mechanism responsible for the origin of small neutrino masses is the so-called seesaw mechanism, by which one can generate Majorana masses of three active neutrinos with renormalizable operators at the tree level. There are three different ways to realize the tree level seesaw mechanism, categorized as Type I [11], Type II [12] and Type III [13] seesaw mechanisms, which can all be naturally embedded into more fundamental frameworks such as the grand unified theories (GUTs) or string theory. The simplest and well-studied mechanism for generating neutrino masses is the Type I seesaw, in which at least two right-handed neutrinos are introduced. In this model, experimental light neutrino masses are explained by the ratio of electroweak scale to large Majorana mass term $\left(M_{N}\right)$ which is a suppression factor, given by $Y_{\nu}^{2} v_{0}^{2} / M_{N}$. The non-ambiguous test of the nature of Majorana neutrinos, and thus a possible test of the seesaw mechanism, will be the observation of the lepton number violating (LNV) processes. Since the CERN Large Hadron Collider (LHC) is going to lead us to a new energy frontier, searching for the heavy Majorana neutrinos at the LHC appears to be very appealing. The ideal production channel to study the LNV processes of heavy Majorana neutrino at hadron collider is the Drell-Yan process via SM gauge boson, $p p \rightarrow W^{*} \rightarrow \ell N$. However, due to the rather small mixing between the heavy neutrinos and the SM leptons in minimal Type I seesaw, to the order $\mathcal{O}\left(m_{\nu} / M_{N}\right)$, the predicted effects of lepton number violation are unlikely to be observable.

The possibility of testing the heavy Majorana neutrino at the LHC beyond the minimal Type I seesaw has been considered by several groups. Recently one group (including one of us) explored the $U(1)_{B-L}$ extension of SM [14]. It is well known that $B-L$ is an accidental global symmetry in the SM and its origin is unknown. In order to understand the origin of Majorana neutrino masses it is crucial to look for new scenarios where $B-L$ is a local symmetry and can be spontaneously broken. The inclusion of three right-handed neutrinos provides an anomaly-free formulation for the gauged $U(1)_{B-L}$. The new gauge boson $Z_{B-L}$ can be produced at the LHC via its gauge interactions with the quarks. Its subsequent decay into a pair of heavy Majorana neutrinos leads to a large sample of events without involving the small mixing suppression. The $\Delta L=2$ signal $p p \rightarrow Z^{\prime} \rightarrow N N \rightarrow \ell^{ \pm} \ell^{ \pm} W^{\mp} W^{\mp}$ with two $W$ bosons hadronic decay will directly test the nature of heavy Majorana neutrinos. However, because no stringent experiments constrain the mass of heavy Majorana neutrino up to now, a pessimistic case with heavy Majorana neutrino mass larger than half of $Z_{B-L}$ boson mass $M_{N}>M_{Z^{\prime}} / 2$ may likely happen in the reality. Consequently one cannot see any lepton number violating signatures at hadron collider because they are kinematically forbidden. This dangerous situation motivates us to figure out other neutrino mass generation 
mechanisms which provide observable signatures in the $U(1)_{B-L}$ extension framework.

On the other hand, if one introduces only two types of fields, scalar $\eta$ and/or fermion $\psi$, neutrino masses can be generated at loop level [15 18]. A salient feature of such radiative seesaw mechanisms is that all the new particles can lie below several TeVs naturally and their interactions with SM particles are not heavily constrained by electroweak precision measurements so that they can be directly tested at the LHC. Other discrete symmetries, for instance $Z_{2}$ symmetry, can be imposed by hand to forbid tree level neutrino mass term if new scalar $\eta$ and fermion $\psi$ stay in the same representation as the scalar doublet in $\mathrm{SM}$ or the scalar triplet $\Delta \sim(1,3,1)$ in Type II seesaw, fermionic singlets $N \sim(1,1,0)$ in Type I seesaw or fermionic triplets $\Sigma \sim(1,3,0)$ in Type III seesaw respectively. Consequently the new particles inside the loop only self-interact and couple to SM leptons, and the lightest one is stable which can serve as cold dark matter candidate. This feature makes it possible to relate the dark matter with neutrino physics. If we restrict our attention to radiative seesaw extended by an extra Abelian gauge symmetry or new representation of other non-Abelian gauge groups, for instance $U(1)_{B-L}$ extension or adjoint representation of $S U(3)$ [19], such feature can also be achieved.

In this paper we investigate a particular realization of a radiative seesaw model in the $U(1)_{B-L}$ extension of SM. In the exact $U(1)_{B-L}$ extension of SM, three right-handed neutrinos $N_{i}$ are introduced and a new scalar field $\Phi$ is added to break the local $B-L$ symmetry. Besides this framework, we add an extra scalar doublet $\eta^{T}=\left(\eta^{+}, \eta^{0}\right)$ and two fermions $\psi_{i}$ in which $\eta$ has no vacuum expectation value. The $B-L$ charges of $\eta, \psi_{i}$ and $\Phi$ are $+1,0$ and +2 , respectively. In this model, there are two terms contributing to neutrino masses: tree level term coming from the modified Type I seesaw mechanism with right-handed neutrino masses generated after $U(1)_{B-L}$ symmetry breaking, and one-loop level term mediated by the new scalar $\eta$ and fermion $\psi_{i}$. The constraints from lepton flavor violating processes are also studied. We focus on $m_{\psi}<m_{\eta}$ scenario in which the lighter $\psi$ could serve as the cold dark matter candidate. Relevant cosmological constraints are investigated. Due to the existence of $U(1)_{B-L}$ gauge symmetry, charged scalar $\eta^{ \pm}$and dark matter $\psi$ have clear signatures associated with SM charged leptons at the LHC: $p p \rightarrow Z^{\prime} \rightarrow \eta^{+} \eta^{-} \rightarrow \ell^{+} \ell^{-} \psi \psi$, even if heavy Majorana neutrinos are forbidden to be produced. We find encouraging results for the LHC signatures of this model to learn about the light neutrino properties and to well-determine the masses of dark matter $\psi$ and its parent particle $\eta$ in such a production topology.

This work is organized as follows: In Section II, we describe our model for neutrino masses and dark matter. In Section III, we discuss the constraints on the neutrino mass and mixing parame- 


\begin{tabular}{|c|c|c|c|c|c||c|c|}
\hline & $Q_{L}, u_{R}, d_{R}$ & $l_{L}, \ell_{R}$ & $N_{R}$ & $H$ & $\Phi$ & $\psi$ & $\eta$ \\
\hline$B-L$ & $\frac{1}{3}$ & -1 & -1 & 0 & +2 & 0 & +1 \\
\hline
\end{tabular}

TABLE I: Fields and their $B-L$ charges in our model, where $\ell=e, \mu, \tau$. The fields on the left-handed side of the double-vertical lines are the exact contents of minimal local $U(1)_{B-L}$ extension of SM.

ters from the current neutrino oscillation data, lepton flavor violating processes and cosmological observation. Section IV is devoted to discuss the possibility of detecting the model at the LHC. We summarize our findings in Section V. Feynman rules for new particles are presented in the Appendix.

\section{THE NEUTRINO MASS AND DARK MATTER MODEL}

In our model the SM is extended with three right-handed Majorana neutrinos $N_{i} \sim(1,1,0)$, one scalar singlet $\Phi \sim(1,1,0)$, one scalar doublet $\eta \sim(1,2,1 / 2)$ and two right-handed Majorana fermions $\psi_{i} \sim(1,1,0)$, as well as $U(1)_{B-L}$ gauge symmetry. The $B-L$ charges for all fields are collected in Table. [1.

With well-chosen charges, as a result, the new lagrangian can be written as

$$
\mathcal{L}=\mathcal{L}_{\text {Kinetic }}+\mathcal{L}_{\text {Scalar }}-\left[Y_{\psi} \overline{\bar{l}_{L}} \widetilde{\eta} \psi_{R}+Y_{\nu} \overline{\bar{l}_{L}} \widetilde{H} N_{R}+\frac{1}{2} m_{\psi} \overline{\psi_{R}^{C}} \psi_{R}+\frac{1}{2} Y_{N} \overline{N_{R}^{C}} N_{R} \Phi+\text { h.c. }\right]
$$

where $l_{L}=\left(\nu_{L}, \ell_{L}\right)$ and $H^{T}=\left(H^{+}, H^{0}\right)$ are the left-handed lepton doublet and scalar doublet in

SM respectively with $\widetilde{H}(\widetilde{\eta})=i \sigma_{2} H^{*}\left(\eta^{*}\right)$. The kinetic term for the matter fields and Lagrangian for scalar fields are

$$
\begin{aligned}
\mathcal{L}_{\text {Kinetic }} & =i \overline{Q_{L}} \gamma^{\mu} D_{\mu} Q_{L}+i \overline{u_{R}} \gamma^{\mu} D_{\mu} u_{R}+i \overline{d_{R}} \gamma^{\mu} D_{\mu} d_{R}+i \overline{l_{L}} \gamma^{\mu} D_{\mu} l_{L}+i \overline{\ell_{R}} \gamma^{\mu} D_{\mu} \ell_{R}+i \overline{N_{R}} \gamma^{\mu} D_{\mu} N_{R} \\
& +i \overline{\psi_{R}} \gamma^{\mu} D_{\mu} \psi_{R}, \\
\mathcal{L}_{\text {Scalar }} & =\left(D_{\mu} H\right)^{\dagger}\left(D^{\mu} H\right)+\left(D_{\mu} \eta\right)^{\dagger}\left(D^{\mu} \eta\right)+\left(D_{\mu} \Phi\right)^{\dagger}\left(D^{\mu} \Phi\right)-V(H, \eta, \Phi)
\end{aligned}
$$

where

$$
D_{\mu} N_{R}=\partial_{\mu} N_{R}-i g_{B L} B_{\mu}^{\prime} N_{R}, D_{\mu} \psi_{R}=\partial_{\mu} \psi_{R}, D_{\mu} \Phi=\partial_{\mu} \Phi+i 2 g_{B L} B_{\mu}^{\prime} \Phi
$$

Here $B_{\mu}^{\prime}$ and $g_{B L}$ are the gauge field and gauge coupling for $U(1)_{B-L}$ respectively. The Higgs potential is given by

$$
V(H, \eta, \Phi)=-m_{H}^{2} H^{\dagger} H-m_{\eta}^{2} \eta^{\dagger} \eta-m_{\Phi}^{2} \Phi^{\dagger} \Phi+\lambda_{H}\left(H^{\dagger} H\right)^{2}+\lambda_{\eta}\left(\eta^{\dagger} \eta\right)^{2}+\lambda_{\Phi}\left(\Phi^{\dagger} \Phi\right)^{2}
$$




$$
\begin{aligned}
& +\lambda_{1}\left(H^{\dagger} H\right)\left(\eta^{\dagger} \eta\right)+\lambda_{2}\left(H^{\dagger} \eta\right)\left(\eta^{\dagger} H\right)+\lambda_{3}\left(H^{\dagger} H\right)\left(\Phi^{\dagger} \Phi\right)+\lambda_{4}\left(\eta^{\dagger} \eta\right)\left(\Phi^{\dagger} \Phi\right) \\
& +\frac{\lambda_{5}}{\Lambda}\left[\left(H \eta^{\dagger}\right)^{2} \Phi+\text { h.c. }\right]
\end{aligned}
$$

where $\lambda_{5}$ has been set to be real without losing generality and $\Lambda$ is a new high energy scale. The last term is a dimension-5 effective operator in this model which has an accidental $B-L$ symmetry. Because of $B-L$ gauge invariance, new fermions $\psi_{i}$ only couple to the new scalar doublet $\eta$ and right-handed neutrinos only couple to SM Higgs $H$. For $m_{H}^{2}, m_{\Phi}^{2}>0$ and $m_{\eta}^{2}<0$, only $H$ and $\Phi$ acquire nonzero vacuum expectation values (VEVs). The $U(1)_{B-L}$ symmetry is spontaneously broken by $\mathrm{VEV}$ of $\Phi$. After imposing the conditions of global minimum, one obtains

$$
\begin{aligned}
v_{0}^{2} & =\frac{\lambda_{\Phi} m_{H}^{2}-\lambda_{3} m_{\Phi}^{2}}{\lambda_{H} \lambda_{\Phi}-\lambda_{3}^{2}}, \\
v_{\Phi}^{2} & =\frac{\lambda_{H} m_{\Phi}^{2}-\lambda_{3} m_{H}^{2}}{\lambda_{H} \lambda_{\Phi}-\lambda_{3}^{2}} .
\end{aligned}
$$

We define $H^{0}=\left(h^{0}+v_{0}+i G^{0}\right) / \sqrt{2}, \eta^{0}=\left(\delta^{0}+i F^{0}\right) / \sqrt{2}, \Phi=\left(\phi^{0}+v_{\Phi}+i K^{0}\right) / \sqrt{2}, H^{ \pm}=G^{ \pm}$and $\eta^{ \pm}=\delta^{ \pm 1}$, and the mass eigenvalues of the resulting physical bosons are given by

- CP-even states:

$$
\begin{aligned}
& h^{0}, m_{h^{0}}^{2}=2 \lambda_{H} v_{0}^{2} \\
& \phi^{0}, m_{\phi^{0}}^{2}=2 \lambda_{\Phi} v_{\Phi}^{2} \\
& \delta^{0}, m_{\delta^{0}}^{2}=-m_{\eta}^{2}+\frac{1}{2}\left(\lambda_{1}+\lambda_{2}\right) v_{0}^{2}+\frac{1}{2} \lambda_{4} v_{\Phi}^{2}+\frac{v_{0}^{2} v_{\Phi} \lambda_{5}}{\sqrt{2} \Lambda}
\end{aligned}
$$

- CP-odd state:

$$
F^{0}, m_{F^{0}}^{2}=-m_{\eta}^{2}+\frac{1}{2}\left(\lambda_{1}+\lambda_{2}\right) v_{0}^{2}+\frac{1}{2} \lambda_{4} v_{\Phi}^{2}-\frac{v_{0}^{2} v_{\Phi} \lambda_{5}}{\sqrt{2} \Lambda}
$$

- charged state:

$$
\delta^{ \pm}, m_{\delta^{ \pm}}^{2}=-m_{\eta}^{2}+\frac{1}{2} \lambda_{1} v_{0}^{2}+\frac{1}{2} \lambda_{4} v_{\Phi}^{2}
$$

- new gauge boson:

$$
Z^{\prime}=Z_{B-L}, M_{Z^{\prime}}=2 g_{B L} v_{\Phi}
$$

\footnotetext{
${ }^{1}$ We denote the new scalar doublet as $\delta$ below.
} 
The mixing angle between $\phi^{0}$ and $h^{0}$ is $\tan 2 \theta=\lambda_{3} v_{0} v_{\Phi} /\left(\lambda_{H} v_{0}^{2}-\lambda_{\Phi} v_{\Phi}^{2}\right)$. Relevant interactions are collected in the Appendix. For simplicity we demand the mass hierarchy in this model as below

$$
\begin{aligned}
& m_{\psi} \equiv m_{\psi_{1}} \lesssim m_{\psi_{2}}<m_{\delta^{ \pm}} \ll m_{\phi^{0}} \sim M_{N} \sim M_{Z^{\prime}} \sim v_{\Phi} \\
& m_{\delta} \equiv m_{\delta^{ \pm}} \approx m_{\delta^{0}} \approx m_{F^{0}} .
\end{aligned}
$$

After $\Phi$ is set at its VEV, right-handed Majorana neutrinos acquire masses, $M_{N}=Y_{N} v_{\Phi} / \sqrt{2}$. According to Eq. (2), there are two terms contributing to light Majorana neutrino mass matrix: tree level Type I seesaw terms $Y_{\nu} \overline{l_{L}} \widetilde{H} N_{R}, \frac{1}{2} Y_{N} \overline{N_{R}^{C}} N_{R} \Phi$ and one-loop radiative corrections as depicted in Fig. 1. Notice that Fig. 1 is induced by the Yukawa coupling among $\psi_{i}, \delta$ and SM leptons and the existence of $\lambda_{5}$ term in Eq. (6) . If $\lambda_{5}$ is zero, neutrino masses would purely come from Type I seesaw term. In the most general case, active neutrino mass matrix can be written as

$$
\left(M_{\nu}\right)_{\alpha \beta}=\left(M_{\text {tree }}\right)_{\alpha \beta}+\left(M_{\text {loop }}\right)_{\alpha \beta}=-\left(M_{D} M_{N}^{-1} M_{D}^{T}\right)_{\alpha \beta}+\sum_{i=1,2}\left(Y_{\psi}\right)_{\alpha i}\left(Y_{\psi}\right)_{\beta i} \frac{I\left(m_{\psi_{i}}^{2} / m_{\delta}^{2}\right)}{m_{\psi_{i}}}
$$

where $M_{D}=Y_{\nu} v_{0} / \sqrt{2}$ is the Dirac neutrino mass matrix and

$$
I(x)=\frac{\lambda_{5} v_{\Phi} v_{0}^{2}}{8 \sqrt{2} \pi^{2} \Lambda}\left(\frac{x}{1-x}\right)\left[1+\frac{x \ln x}{1-x}\right] .
$$

Neutrino mass formula in Eq. (16) is called as the "hybrid" seesaw mechanism. There are three scenarios: (1) $\mathcal{O}\left(M_{\text {tree }}\right) \gg \mathcal{O}\left(M_{\text {loop }}\right)$, Type I seesaw term dominates the contribution to the neutrino masses; (2) $\mathcal{O}\left(M_{\text {tree }}\right) \sim \mathcal{O}\left(M_{\text {loop }}\right)$, both terms contribute to neutrino masses; and (3) $\mathcal{O}\left(M_{\text {tree }}\right) \ll \mathcal{O}\left(M_{\text {loop }}\right)$, radiative seesaw term dominates. We can impose $Z_{2}$ discrete symmetry for right-handed neutrino $N_{R} \rightarrow-N_{R}$ to eliminate the Type I seesaw term, but actually the detectable phenomenology discussed later does not depend on which scenario we work on. Because the pure Type I seesaw scenario (1) has been well-studied by many groups both theoretically and phenomenologically, we mainly concentrate our scope on the other extreme scenario (3) in Section 3.

It is important to emphasize that the last term in Eq. (6) is not the only dimension-5 effective operator in this model. There are other dimension-5 operators allowed by gauge symmetry, for instance $\frac{1}{\Lambda}\left(H^{\dagger} H+\eta^{\dagger} \eta+\Phi^{\dagger} \Phi\right) \overline{\psi_{R}^{C}} \psi_{R}, \frac{1}{\Lambda} H^{\dagger} \eta \psi_{R} N_{R}, \frac{1}{\Lambda} l_{L}^{T} H \Phi N_{R}, \frac{1}{\Lambda} l_{L} l_{L} \eta \eta$. Fermion $\psi$ can get additional but suppressed mass contribution $\left(v_{0}^{2}+v_{\Phi}^{2}\right) / \Lambda \cdot \frac{1}{\Lambda} H^{\dagger} \eta \psi_{R} N_{R}$ term contributes to right-handed neutrino mass to the order $v_{0}^{2} / \Lambda^{2}$ and $\frac{1}{\Lambda} l_{L}^{T} H \Phi N_{R}$ contributes to Dirac neutrino mass term to the order $v_{\Phi} / \Lambda . \frac{1}{\Lambda} l_{L} l_{L} \eta \eta$ can give extra neutrino mass term $\left(m_{\psi} / \Lambda\right) M_{\text {loop }}$ in Eq. (16). In brief, all other dimension-5 operators supply additional suppression factor for neutrino mass generation or 


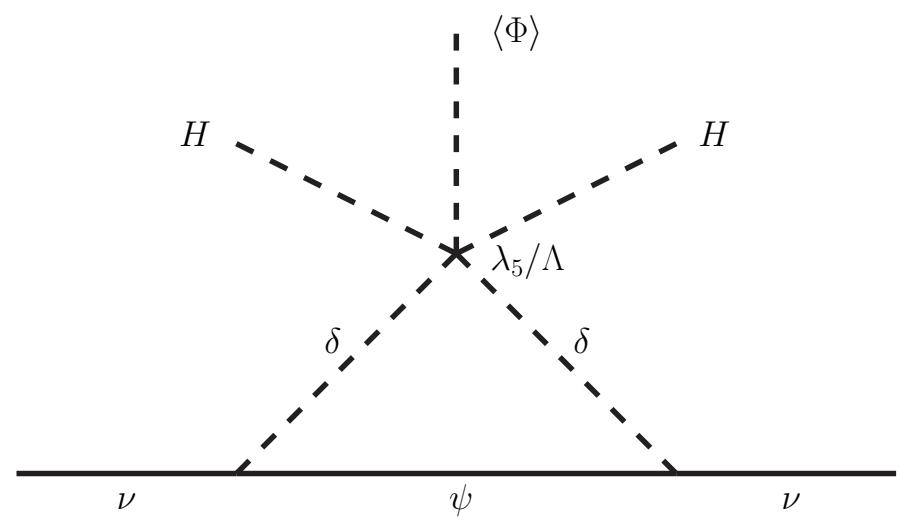

FIG. 1: Feynman diagram for loop-level neutrino mass generation in this model.

lepton flavor violation. We therefore ignore these suppressed contributions in our analysis. The usual neutrino seesaw mass term $\frac{1}{\Lambda} l_{L} l_{L} H H$ is forbidden by $U(1)_{B-L}$ gauge symmetry.

Notice that the new particles $\psi, \delta$ inside the loop only couple to SM leptons and do not have mass mixing terms like $M_{D}$. Therefore another emergent consequence of this model is the appearance of a lightest stable particle, which can serve as the cold dark matter. It can be bosonic [20] (i.e. the lighter one of $\delta^{0}$ and $F^{0}$ ) or fermionic [21 23]] (i.e. the lighter one of $\psi_{i}$ ). In this paper, as shown in Eq. (15), we assume $m_{\psi}<m_{\delta}$ and two fermions $\psi_{1}, \psi_{2}$ are quasi-degenerate. Consequently the lighter $\psi$ would be the cold dark matter candidate.

\section{CONSTRAINT ON THE PARAMETER SPACE}

In this section, we discuss the constraints on the parameter space of the model from (a) neutrino physics, (b) lepton flavor violating processes and (c) cosmological observation.

\section{A. Neutrino Masses from Radiative Seesaw}

We assume that there is only $M_{\text {loop }}$ term left in Eq. (16). The neutrino mass matrix can be diagonalized as

$$
\hat{M}=V_{P M N S}^{\dagger} M_{\nu} V_{P M N S}^{*}
$$

where $\hat{M}=\operatorname{diag}\left(m_{1}, m_{2}, m_{3}\right) . \quad V_{P M N S}$ is the lepton mixing matrix, i.e. the Pontecorvo-MakiNakagawa-Sakata (PMNS) matrix [24], which comes from the mismatch between the diagonalization of neutrino masses and charged lepton mass matrix. With the standard parametrization, 
$V_{P M N S}$ can be written as

$$
V_{P M N S}=\left(\begin{array}{lll}
c_{12} c_{13} & c_{13} s_{12} & e^{-\mathrm{i} \delta} s_{13} \\
-c_{12} s_{13} s_{23} e^{\mathrm{i} \delta}-c_{23} s_{12} & c_{12} c_{23}-e^{\mathrm{i} \delta} s_{12} s_{13} s_{23} & c_{13} s_{23} \\
s_{12} s_{23}-e^{\mathrm{i} \delta} c_{12} c_{23} s_{13} & -c_{23} s_{12} s_{13} e^{\mathrm{i} \delta}-c_{12} s_{23} & c_{13} c_{23}
\end{array}\right) \times \operatorname{diag}\left(1, e^{i \chi}, 1\right)
$$

where $s_{i j}=\sin \theta_{i j}, c_{i j}=\cos \theta_{i j}, 0 \leq \theta_{i j} \leq \pi / 2$ and $0 \leq \delta, \chi \leq 2 \pi$. The phase $\delta$ is the Dirac CP phase, and $\chi$ is the Majorana phase. The experimental constraints on the neutrino masses and mixing angles, at $2 \sigma$ level are [25]

$$
\begin{aligned}
7.25 \times 10^{-5} \mathrm{eV}^{2} & <\Delta m_{21}^{2}<8.11 \times 10^{-5} \mathrm{eV}^{2} \\
2.18 \times 10^{-3} \mathrm{eV}^{2} & <\left|\Delta m_{31}^{2}\right|<2.64 \times 10^{-3} \mathrm{eV}^{2} \\
0.27< & \sin ^{2} \theta_{12}<0.35 \\
0.39< & \sin ^{2} \theta_{23}<0.63 \\
\sin ^{2} \theta_{13} & <0.04
\end{aligned}
$$

and $\sum_{i} m_{i}<1.2 \mathrm{eV}$. Using Casas-Ibarra parametrization [26], one can find a formal solution for the Yukawa coupling between the SM charged leptons $(\ell=e, \mu, \tau)$ and $\psi_{i}(i=1,2)$

$$
\left(Y_{\psi}\right)_{\ell i}=V_{P M N S} \hat{M}^{1 / 2} O F^{-1 / 2}
$$

in terms of a complex matrix which satisfies the orthogonality condition $O^{T} O=O O^{T}=1$, where $F=\operatorname{diag}\left(I\left(m_{\psi_{1}}^{2} / m_{\delta}\right) / m_{\psi_{1}}, I\left(m_{\psi_{2}}^{2} / m_{\delta}\right) / m_{\psi_{2}}\right)$. In the $3+2$ mode, one of the active neutrinos is massless and $\hat{M}=\operatorname{diag}\left(0, \sqrt{\Delta m_{21}^{2}}, \sqrt{\left|\Delta m_{31}^{2}\right|}\right)$ for normal hierarchy $(\mathrm{NH}), \hat{M}=$ $\operatorname{diag}\left(\sqrt{\left|\Delta m_{31}^{2}\right|}, \sqrt{\Delta m_{21}^{2}+\left|\Delta m_{31}^{2}\right|}, 0\right)$ for inverted hierarchy (IH). The matrix $O$ can be written as [27]

$$
O=\left(\begin{array}{cc}
0 & 0 \\
\cos z & \sin z \\
-\sin z & \cos z
\end{array}\right) \text { for } \mathrm{NH}, O=\left(\begin{array}{cc}
\cos z & \sin z \\
-\sin z & \cos z \\
0 & 0
\end{array}\right) \text { for IH }
$$

where $z=x+i y$ with real parameters $x$ and $y$.

Assuming vanishing Majorana phase and $0 \leq x \leq 2 \pi$, we show $F\left|\left(Y_{\psi}\right)_{\ell 1}\right|^{2} / 1 \mathrm{eV}(\ell=e, \mu, \tau)$ versus parameter $y$ in Fig. 2. The behaviors for $F\left|\left(Y_{\psi}\right)_{\ell 2}\right|^{2} / 1 \mathrm{eV}$ are quite similar. One can see $\mu, \tau$ elements are several times larger than that of electron in $\mathrm{NH}$ and electron element is slightly larger in $\mathrm{IH}$. 

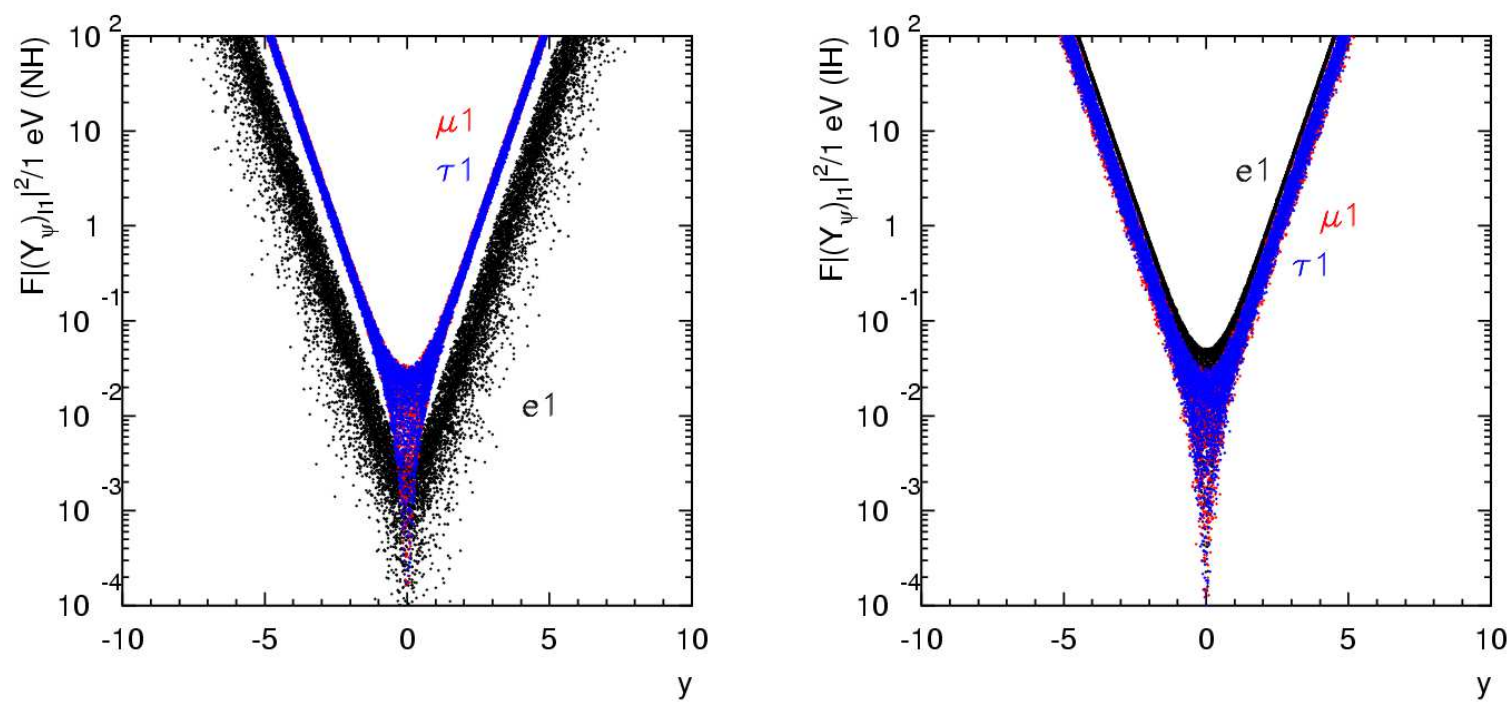

FIG. 2: $F\left|\left(Y_{\psi}\right)_{\ell 1}\right|^{2} / 1 \mathrm{eV}$ versus parameter $y$ in matrix $O$ for $\mathrm{NH}$ (left) and IH (right), assuming vanishing Majorana phase and $0 \leq x \leq 2 \pi$.

\section{B. Constraints from Lepton Flavor Violating Process}

Here we briefly discuss constraints on the parameter space from lepton flavor violating (LFV) processes such as $\mu \rightarrow e \gamma$. One-loop diagrams mediated by $\eta^{ \pm}$and $\psi$, similar to the one generating small neutrino masses, contribute to the radiative decay processes. The branching ratio of $\mu \rightarrow e \gamma$ decay was discussed in Refs. [21 23]

$$
B R(\mu \rightarrow e \gamma)=\frac{3 \alpha}{64 \pi\left(G_{F} m_{\delta}^{2}\right)^{2}}\left|\sum_{i}\left(Y_{\psi}\right)_{\mu i}\left(Y_{\psi}\right)_{e i}^{*} F_{2}\left(m_{\psi_{i}}^{2} / m_{\delta}^{2}\right)\right|^{2}
$$

where

$$
F_{2}(x)=\frac{1-6 x+3 x^{2}+2 x^{3}-6 x^{2} \ln x}{6(1-x)^{4}} .
$$

In principle, we can always tune the function of parameter $\lambda_{5}$ to get appropriate Yukawa couple $Y_{\psi}$ to escape from lepton flavor violating constraints. In Fig. 3 we show the branching fraction of $\mu \rightarrow e \gamma$ versus parameter $y$ by assuming $0 \leq x \leq 2 \pi, F=1 \mathrm{eV}, m_{\psi}=100 \mathrm{GeV}$ and $m_{\delta}=300 \mathrm{GeV}$. The horizontal line is the current experimental bound, i.e. $B R(\mu \rightarrow e \gamma)<1.2 \times 10^{-11}$ [28]. One can find that $\mu \rightarrow e \gamma$ restricts $|y|$ to be less than 3 for $\mathrm{NH}$ and 2 for $\mathrm{IH}$. Combining the constraints from neutrino masses as shown in Fig. 2 with LFV in Fig. 3, we have Yukawa couplings as $\left|\left(Y_{\psi}\right)_{e 1}\right|^{2} \lesssim 0.5,\left|\left(Y_{\psi}\right)_{\mu 1, \tau 1}\right|^{2} \lesssim 1$ for $\mathrm{NH}$ and $\left|\left(Y_{\psi}\right)_{e 1, \mu 1, \tau 1}\right|^{2} \lesssim 1$ for IH, which are not stringently constrained. 

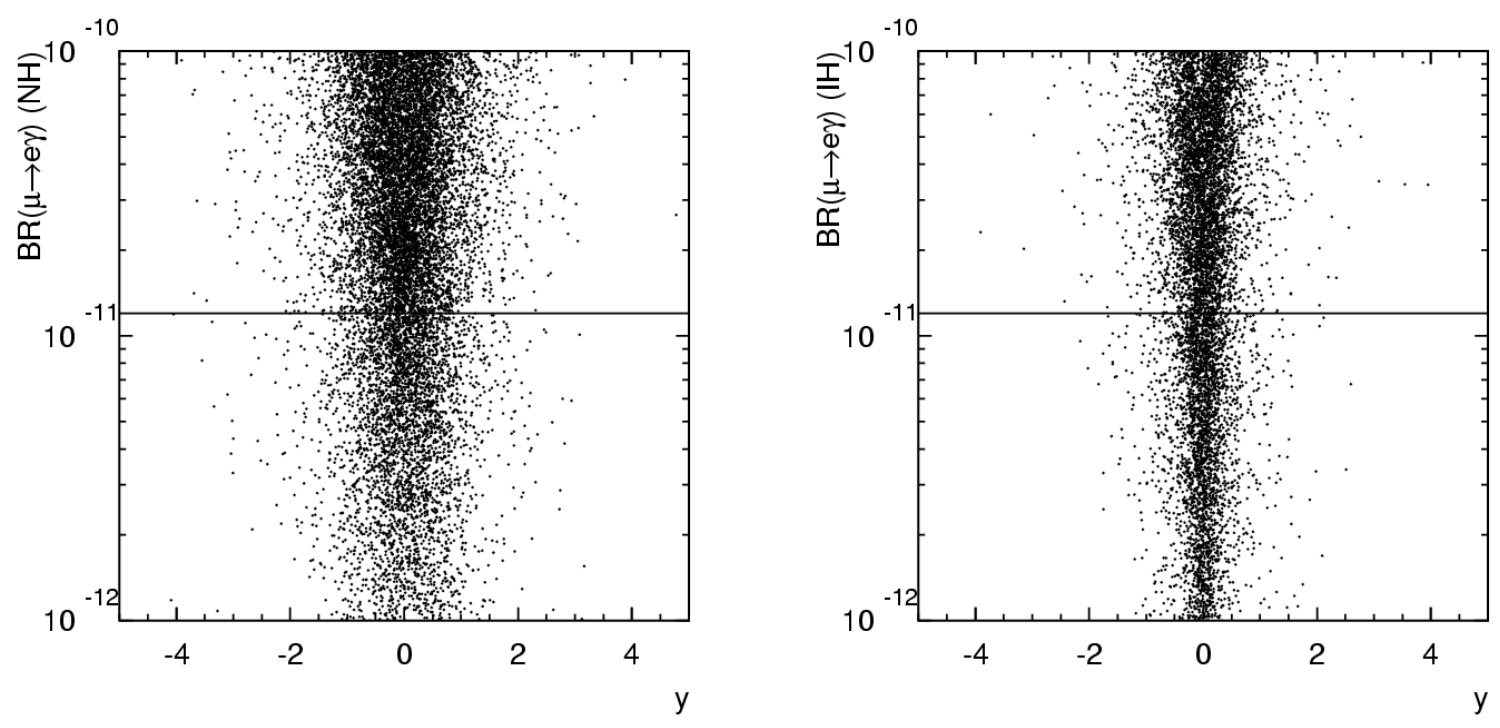

FIG. 3: The branching fraction of $\mu \rightarrow e \gamma$ versus parameter $y$ in matrix $O$ for NH (left) and IH (right), assuming vanishing Majorana phase, $0 \leq x \leq 2 \pi, F=1 \mathrm{eV}, m_{\psi}=100 \mathrm{GeV}$ and $m_{\delta}=300 \mathrm{GeV}$. The horizontal line represents the current experimental bound for $B R(\mu \rightarrow e \gamma)<1.2 \times 10^{-11}$.

\section{Cosmological Constraint}

Because of the mass hierarchy assumed in previous section, the lighter one of $\psi_{i}$ would be the dark matter candidate in this model, denoted as $\psi$. Its relic density is calculable as a function of Yukawa coupling $Y_{\psi}$, dark matter mass $m_{\psi}$ and mass of scalar $\delta$. In this model, the relic density of $\psi$ is controlled by the annihilation of $\psi \psi \rightarrow \nu \nu, \ell^{+} \ell^{-}$through the exchange of $\delta^{ \pm}, \delta^{0}$ and $F^{0}$ in t-channel. Because the processes through $\delta^{0}$ and $F^{0}$ exchanges have huge cancellation, two $\psi$ 's annihilate mainly into two charged leptons. Ignoring charged lepton masses, one can write down the interaction rate $\sigma v$ in non-relativistic limit $[21$ 23]

$$
\sigma_{a n n} v_{r e l} \equiv a+b v_{r e l}^{2}=\sum_{\alpha \beta}\left|\left(Y_{\psi}\right)_{\alpha 1}\right|^{2}\left|\left(Y_{\psi}\right)_{\beta 1}\right|^{2_{\psi}^{2}} \frac{r_{\psi}\left(1-2 r_{\psi}+2 r_{\psi}^{2}\right)}{24 \pi m_{\psi}^{2}} v_{r e l}^{2},
$$

where $r_{\psi}=m_{\psi}^{2} /\left(m_{\psi}^{2}+m_{\delta}^{2}\right)$ and $v_{r e l}$ is relative speed. The thermally averaged cross section can be written as $\left\langle\sigma_{\text {anna }} v_{\text {rel }}\right\rangle=a+6 b / x_{f}$, where $x_{f}=m_{\psi} / T_{f}$ and $T_{f}$ is the freeze-out temperature of the relic particle. The present density of $\psi$ is simply given by $\rho_{\psi}=m_{\psi} s_{0} Y_{\infty}$, where $s_{0}=2889.2 \mathrm{~cm}^{-3}$ is the present entropy density and $Y_{\infty}$ is the asymptotic value of the ratio $n_{\psi} / s_{0}$ with $Y_{\infty}^{-1}=$ $0.264 \sqrt{g_{*}} M_{P l} m_{\psi}\left(a+3 b / x_{f}\right) x_{f}^{-1}$ through the time (temperature) evolution which is obtained by solving the Boltzmann equation. The relic density can finally be expressed in terms of the critical 


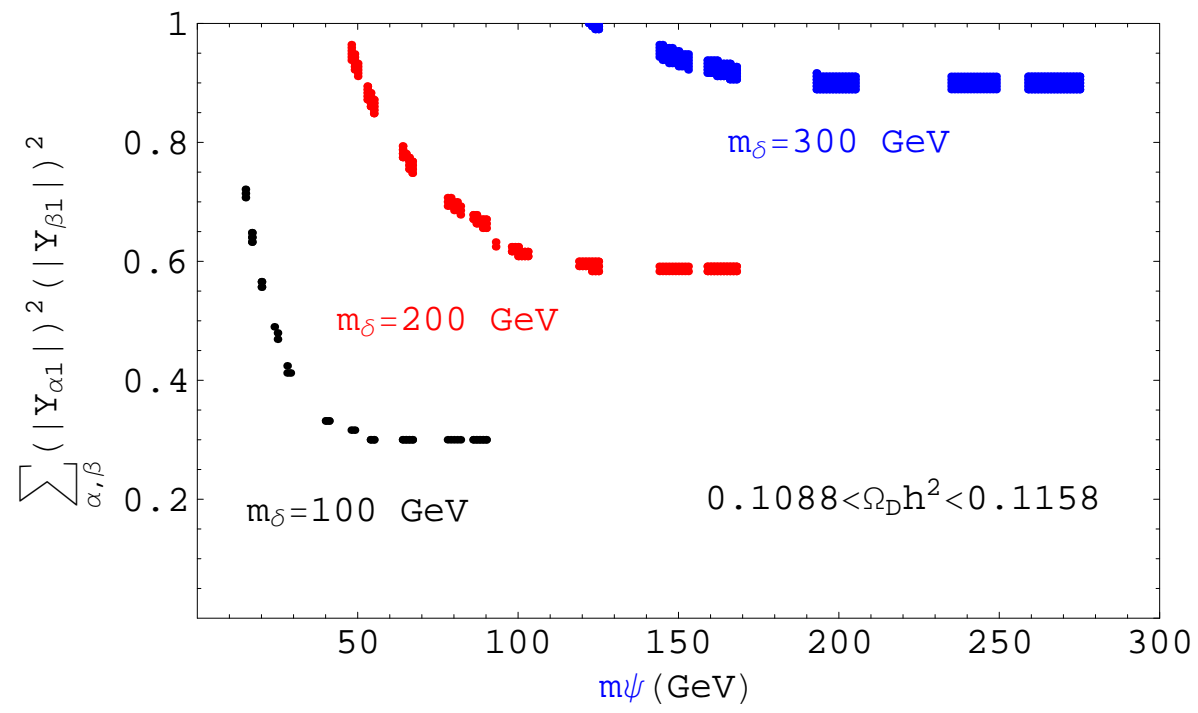

FIG. 4: $\sum_{\alpha \beta}\left|\left(Y_{\psi}\right)_{\alpha 1}\right|^{2}\left|\left(Y_{\psi}\right)_{\beta 1}\right|^{2}$ versus dark matter mass $m_{\psi}$ constrained by dark matter relic density.

density

$$
\Omega_{D} h^{2} \simeq \frac{1.07 \times 10^{9} \mathrm{GeV}^{-1}}{M_{P l}} \frac{x_{f}}{\sqrt{g_{*}}} \frac{1}{a+3 b / x_{f}}
$$

where $h$ is the Hubble constant in units of $100 \mathrm{~km} / \mathrm{s} \cdot \mathrm{Mpc}, M_{P l}=1.22 \times 10^{19} \mathrm{GeV}$ is the Planck mass and $g_{*}$ is the number of relativistic degrees of freedom with mass less than $T_{f}$. The freeze-out temperature $x_{f}$ can be estimated through the iterative solution of the equation [29]

$$
x_{f}=\ln \left[c(c+2) \sqrt{\frac{45}{8}} \frac{g}{2 \pi^{3}} \frac{M_{P l} m_{\psi}\left\langle\sigma_{a n n} v_{r e l}\right\rangle}{\sqrt{g_{*} x_{f}}}\right] \simeq \ln \frac{0.038 M_{P l} m_{\psi}\left(a+6 b / x_{f}\right)}{\sqrt{g_{*} x_{f}}}
$$

where $c$ is the constant of order one determined by matching the late-time and early-time solutions and $g$ is the weak interaction gauge coupling constant.

In Fig. 4 we show $\sum_{\alpha \beta}\left|\left(Y_{\psi}\right)_{\alpha 1}\right|^{2}\left|\left(Y_{\psi}\right)_{\beta 1}\right|^{2}$ versus dark matter mass $m_{\psi}$ constrained by dark matter relic density with different $\delta^{ \pm}$masses $m_{\delta}=100 \mathrm{GeV}, 200 \mathrm{GeV}, 300 \mathrm{GeV}$. We find, to get correct relic density, Yukawa coupling $Y_{\psi}$ should be at order $\mathcal{O}(1)$ with a few hundred $\operatorname{GeV} m_{\psi}$ and $m_{\delta}$.

\section{THE TEST OF RADIATIVE SEESAW IN B-L EXTENSION AT THE LHC}

In order to study the predictions for the lepton flavor correlation and dark matter property with the radiative seesaw mechanism in $U(1)_{B-L}$ extension, the ideal channel is production of new scalar $\delta$ and fermion $\psi$ via the mediation of new gauge boson $Z^{\prime}$ in this theory. 


\section{A. New Gauge Boson $Z^{\prime}$ Decay}

In the limit where there is no mixing between the two Abelian sectors of the minimal $B-L$ extension of the SM, the mass of the new gauge boson $Z^{\prime}$ is given as $M_{Z^{\prime}}=2 g_{B L} v_{\Phi}$, see Eq. (14). To satisfy the experimental lower bound, $M_{Z^{\prime}} / g_{B L}>5-10 \mathrm{TeV}$ [30], it is sufficient to assume that $v_{\Phi}>2.5-5 \mathrm{TeV}$. There has been a lot of works on the heavy neutral gauge bosons. For a recent review, see Ref. [31], and recent studies of $Z^{\prime}$ at the Tevatron and LHC [32]. For a recent study of the phenomenological aspects of the $B-L$ model, see Ref. [33]. In Ref. [14] heavy Majorana neutrino pair production via $Z^{\prime}$ is well-studied when $M_{N}<M_{Z^{\prime}} / 2$.

Here, as indicated in earlier section, we pay attention to the observable signatures in this model when the case $M_{N}>M_{Z^{\prime}} / 2$ happens and thus the pair production of heavy Majorana neutrinos from on-shell $Z^{\prime}$ is forbidden. Therefore, with the mass hierarchy given in Eq. (15), the partial widths of $Z^{\prime}$ decay are

$$
\begin{aligned}
\Gamma\left(Z^{\prime} \rightarrow f \bar{f}\right) & =g_{B L}^{2} \frac{M_{Z^{\prime}}}{12 \pi} C_{f}\left(Q_{B L}^{f}\right)^{2}\left(1+2 \frac{m_{f}^{2}}{M_{Z^{\prime}}^{2}}\right) \beta_{f}, \\
\Gamma\left(Z^{\prime} \rightarrow \sum_{m} \nu_{m} \nu_{m}\right) & =3 g_{B L}^{2} \frac{M_{Z^{\prime}}}{24 \pi} C_{\nu}\left(Q_{B L}^{\ell}\right)^{2}, \\
\Gamma\left(Z^{\prime} \rightarrow \delta^{+} \delta^{-}\right) & =\frac{g_{B L}^{2}}{48 \pi} M_{Z^{\prime}} \beta_{\delta^{ \pm}}^{3}=\frac{g_{B L}^{2}}{48 \pi} M_{Z^{\prime}} \beta_{\delta}^{3}, \\
\Gamma\left(Z^{\prime} \rightarrow \delta^{0} F^{0}\right) & =\frac{g_{B L}^{2}}{48 \pi} M_{Z^{\prime}}\left[1-2 \frac{m_{\delta^{0}}^{2}+m_{F^{0}}^{2}}{M_{Z^{\prime}}^{2}}+\frac{\left(m_{\delta^{0}}^{2}-m_{F^{0}}^{2}\right)^{2}}{M_{Z^{\prime}}^{4}}\right]^{3 / 2}=\frac{g_{B L}^{2}}{48 \pi} M_{Z^{\prime}} \beta_{\delta}^{3}
\end{aligned}
$$

where $f=\ell, q$, the couplings $C_{\ell, \nu}=1, C_{q}=3$, and $\beta_{i}=\sqrt{1-4 m_{i}^{2} / M_{Z^{\prime}}^{2}}$ is the speed of particle $i$. In Fig. 5 we plot the branching ratios of $Z^{\prime}$ decay versus $m_{\delta}$ when $M_{Z^{\prime}}=1 \mathrm{TeV}$. Notice the mass of new scalar $m_{\delta}$ is constrained by dark matter relic density as several hundred GeV. One can see, in low $m_{\delta}$ range, the $Z^{\prime}$ decay branching fractions take simple ratios for the final states

$$
\sum_{\ell}^{e, \mu, \tau} \ell^{+} \ell^{-}: \sum_{q}^{u \cdots t} q \bar{q}: \sum_{m}^{1,2,3} \nu_{m} \nu_{m}: \delta^{+} \delta^{-}: \delta^{0} F^{0}=3: 2: \frac{3}{2}: \frac{1}{4}: \frac{1}{4} .
$$

\section{B. New Charged Scalar $\delta^{ \pm}$Decay}

As seen in previous section, the new scalar $\delta$ only couples to the stable particle $\psi$ and SM leptons because of $B-L$ gauge invariance. The leading decay channels for the new scalar include $\delta^{ \pm} \rightarrow \ell^{ \pm} \psi_{1,2}$ and $\delta^{0} \rightarrow \nu(\bar{\nu}) \psi_{1,2}$. All relevant amplitudes are proportional to the Yukawa coupling $Y_{\psi}$ which contributes to light neutrino mass generation. Therefore the lepton-flavor contents of $\delta$ decay will be different in each neutrino spectrum. 


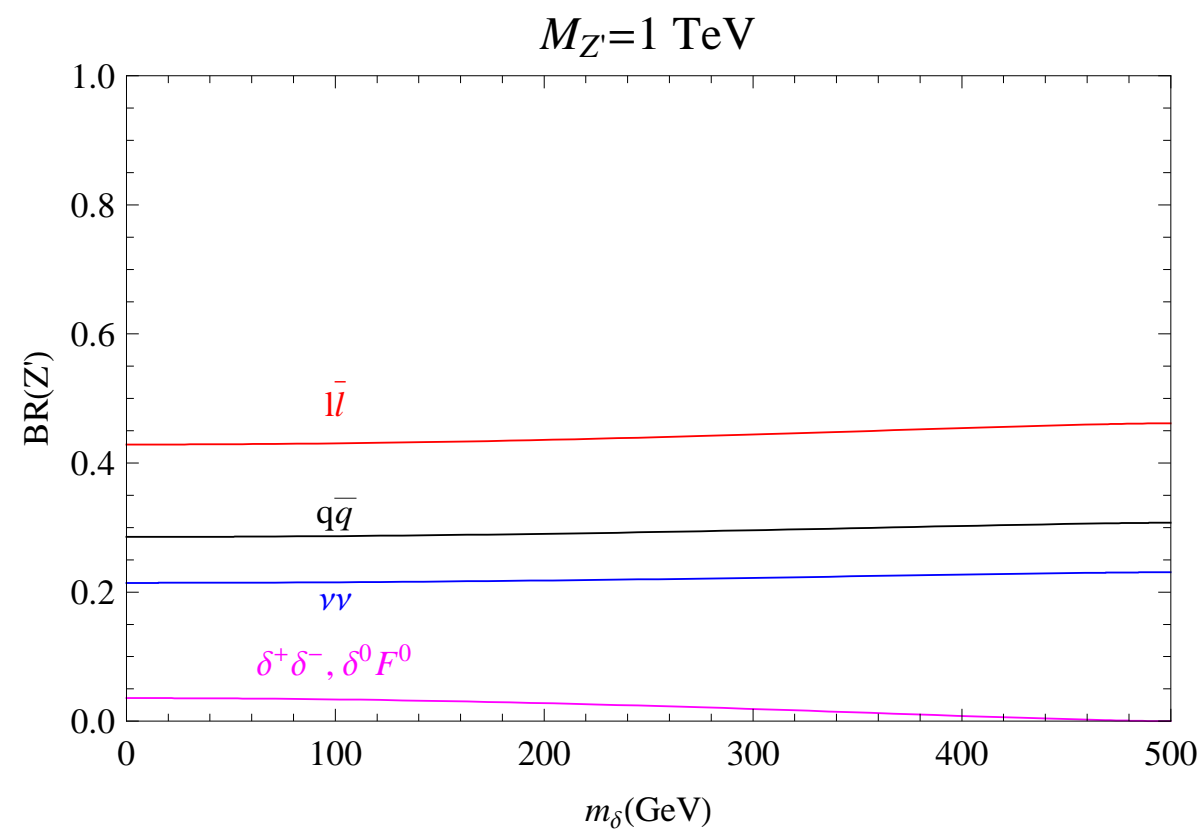

FIG. 5: The branching ratios of $Z^{\prime}$ decay versus $m_{\delta}$ when $M_{Z^{\prime}}=1 \mathrm{TeV}$.

The partial widths of $\delta^{ \pm}$decay are

$$
\Gamma\left(\delta^{ \pm} \rightarrow \ell^{ \pm} \psi_{1(2)}\right)=\frac{\left|\left(Y_{\psi}\right)_{\ell 1(2)}\right|^{2}}{16 \pi m_{\delta^{ \pm}}} \lambda^{1 / 2}\left(1, \frac{m_{\ell}^{2}}{m_{\delta^{ \pm}}^{2}}, \frac{m_{\psi_{1(2)}}^{2}}{m_{\delta^{ \pm}}^{2}}\right)\left(m_{\delta^{ \pm}}^{2}-m_{\ell}^{2}-m_{\psi_{1(2)}}^{2}\right)
$$

where $\lambda(x, y, z)=x^{2}+y^{2}+z^{2}-2 x y-2 x z-2 y z$. The dependence of $\delta^{ \pm}$decay branching fractions $\delta^{ \pm} \rightarrow \ell^{ \pm} \psi_{1}(\ell=e, \mu, \tau)$ on parameter y is plotted in Fig. 6. The behaviors for $\psi_{2}$ final state are almost the same. One can see that if SM charged lepton masses are ignored, the decay branching fractions do not depend on both masses of $\delta$ and $\psi_{1}$ entirely, and the parameter $y$ except for range $|y| \lesssim 1$. The branching fractions can differ by one order of magnitude in $\mathrm{NH}$ case $B R\left(e^{ \pm} \psi_{1}\right) \ll B R\left(\mu^{ \pm} \psi_{1}\right), B R\left(\tau^{ \pm} \psi_{1}\right) \approx 30 \%$ and a few times in the IH spectrum $B R\left(\mu^{ \pm} \psi_{1}\right), B R\left(\tau^{ \pm} \psi_{1}\right)<B R\left(e^{ \pm} \psi_{1}\right) \approx 25 \%$. Therefore one can distinguish neutrino mass spectra according to different SM lepton flavors of dominant channels in final states. In Fig. 7, we show the dependence of $\delta^{ \pm}$decay branching fractions on Majorana phase $\chi$ in NH and IH for $y=2$. In $\mathrm{NH}$ the dominant channels swap from $\tau^{ \pm} \psi_{1}$ when $\chi \approx \pi / 2$ to $\mu^{ \pm} \psi_{1}$ when $\chi \approx 3 \pi / 2$ by a few times. In IH the dominant channels swap from $e^{ \pm} \psi_{1}$ when $\chi \approx \pi / 2$ to $\mu^{ \pm} \psi_{1}, \tau^{ \pm} \psi_{1}$ when $\chi \approx 3 \pi / 2$ by more than one order of magnitude. This qualitative change can be made use of extracting the value of the Majorana phase $\chi$ and parameter $y$. Moreover, it is important to note that the curves of branching fractions corresponding to Majorana phase translate parallelly by a phase $\pi$ for $-y$ case. 

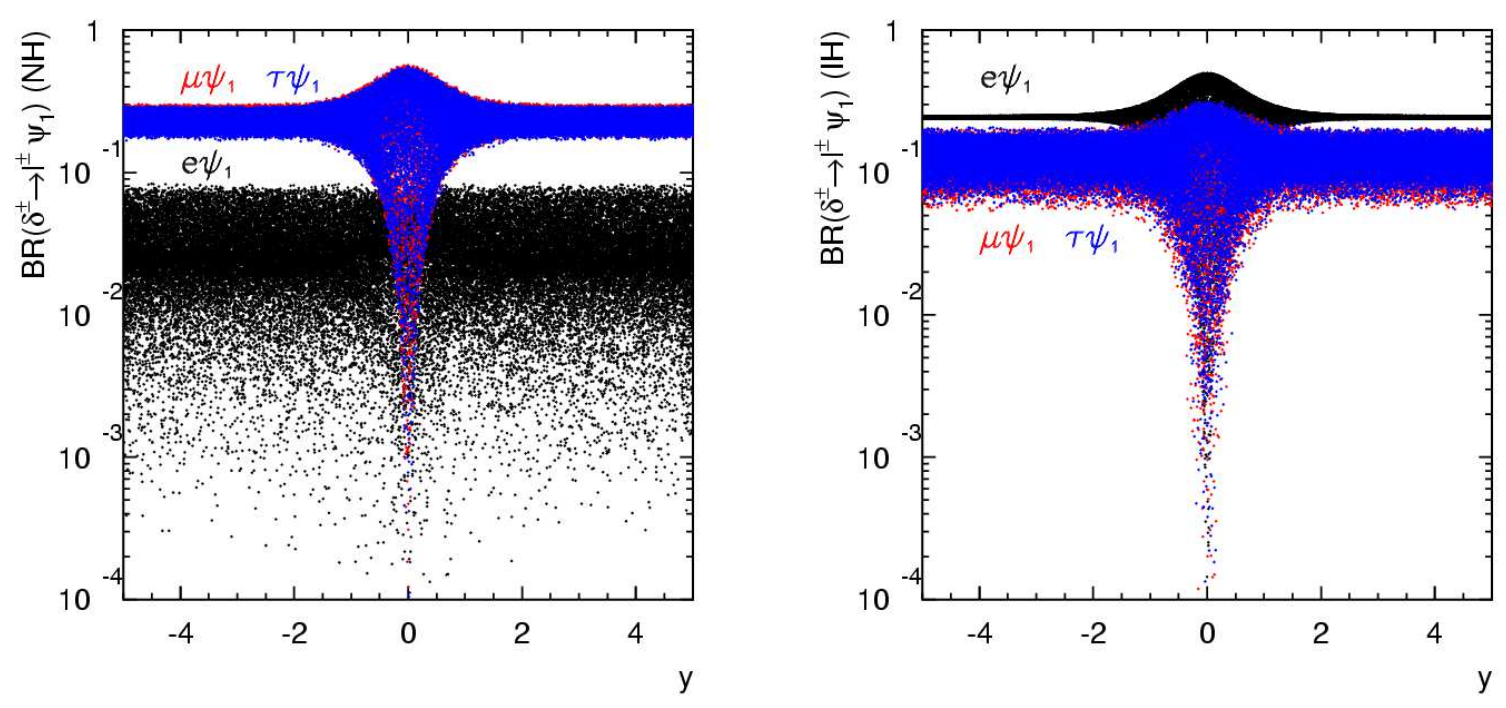

FIG. 6: The branching ratios of $\delta^{ \pm} \rightarrow \ell^{ \pm} \psi_{1}(\ell=e, \mu, \tau)$ versus parameter $y$ in matrix $O$ for NH (left) and IH (right), assuming vanishing Majorana phase, $0 \leq x \leq 2 \pi$.

\section{Production and Mass Determination of Dark Matter $\psi$ and $\delta^{ \pm}$at the LHC}

Since in this model one has a dynamical mechanism for $B-L$ breaking, there is a typical pair production mechanism of $\delta^{ \pm}$through $Z^{\prime}$ with $\delta^{ \pm}$decay into SM charged leptons and the stable particle $\psi$

$$
p p \rightarrow Z^{\prime} \rightarrow \delta^{+} \delta^{-} \rightarrow \ell^{+} \ell^{-} \psi \psi
$$

In Fig. 8 (a) we plot the total cross section of charged scalar $\delta^{ \pm}$pair production at the LHC versus its mass $m_{\delta}$. Considering the mass difference between $\delta^{ \pm}$and $\psi$ can be large, see Fig. 4, our signal would be two hard opposite-sign leptons plus large missing energy. The irreducible SM backgrounds are $Z(\rightarrow \nu \bar{\nu}) Z / \gamma^{*}\left(\rightarrow \ell^{+} \ell^{-}\right)$and $W^{+}\left(\rightarrow \ell^{+} \nu\right) W^{-}\left(\rightarrow \ell^{-} \bar{\nu}\right)$. For our numerical analyzes, we adopt the CTEQ6L1 parton distribution function [34] and evaluate the SM backgrounds by using the automatic package Madgraph [35]. We work in the parton-level, but simulate the detector effects by the kinematical acceptance and employ the Gaussian smearing for the electromagnetic energies [36]

$$
\frac{\Delta E}{E}=\frac{a_{c a l}}{\sqrt{E / G e V}} \oplus b_{c a l}, \quad a_{c a l}=10 \%, b_{c a l}=0.7 \% .
$$

We employ the following basic acceptance cuts for the event selection [36]

$$
p_{T}(\ell) \geq 15 \mathrm{GeV},|\eta(\ell)|<2.5, \Delta R_{\ell \ell} \geq 0.4, \mathscr{E}_{T}>30 \mathrm{GeV}
$$



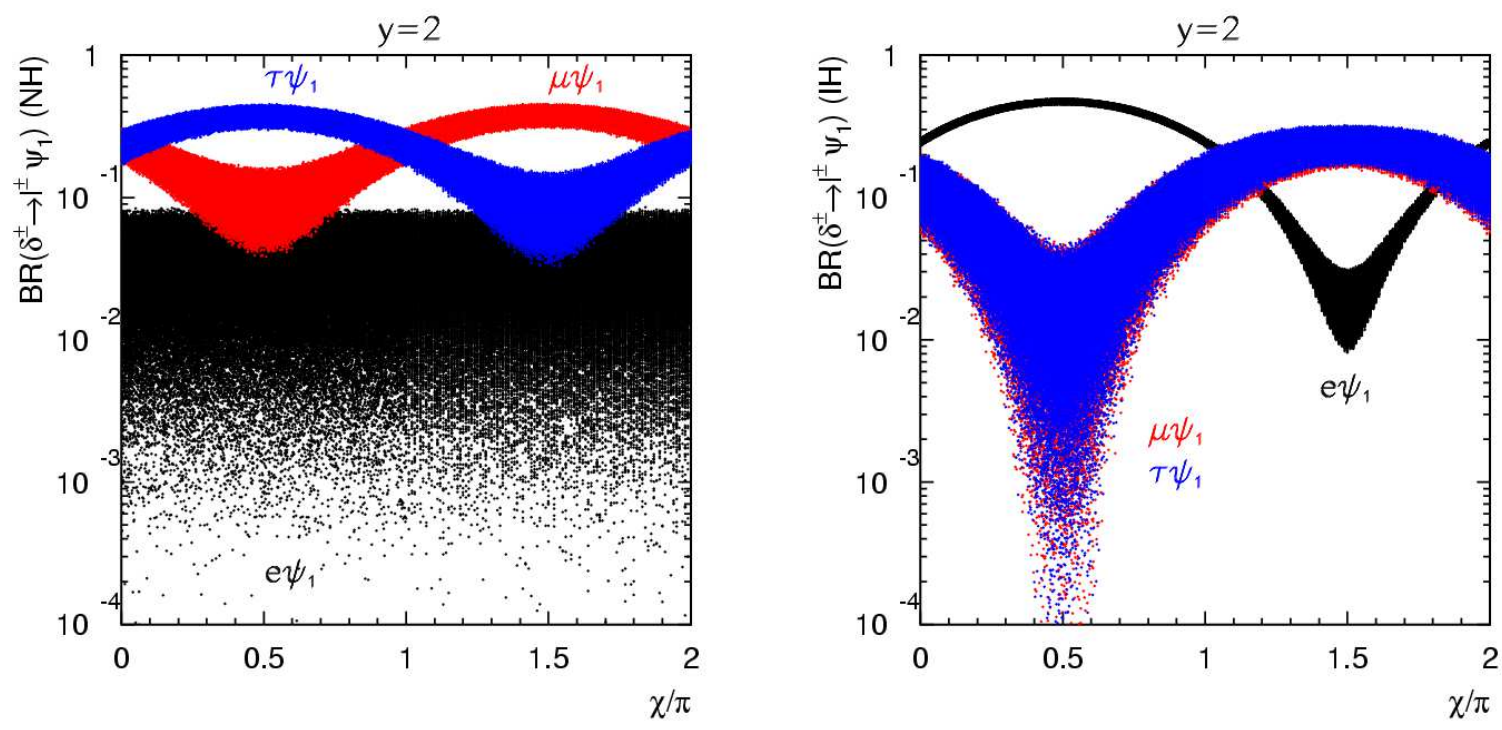

FIG. 7: The branching ratios of $\delta^{ \pm} \rightarrow \ell^{ \pm} \psi_{1}(\ell=e, \mu, \tau)$ versus Majorana phase $\chi$ for NH (left) and IH (right), when $y=2$ and $0 \leq x \leq 2 \pi$.

Because the two leptons can be very hard, we tighten up the transverse momenta of them

$$
p_{T}(\ell)>120 \mathrm{GeV} \text {. }
$$

This cut also helps for the background reduction significantly. The total cross section with all the cuts being set is plotted in Fig. 8 (b), in which we take the branching fraction of $\delta^{ \pm}$as $B R\left(\delta^{ \pm} \rightarrow \ell^{ \pm} \psi\right)=30 \%$ from Fig. 6. For lower mass range of $m_{\delta}$ and $M_{Z^{\prime}}$ one can have $\mathcal{O}(100)$ event number with $100 \mathrm{fb}^{-1}$ integrated luminosity.

On the other hand, the masses of $\psi$ and $\delta^{ \pm}$can be well-determined in this topology with the help of so-called cusp kinematics [37]. Considering the charged leptons in final states to be massless, due to the on-shell constraint for the particle $\delta^{ \pm}$there is a cusp and end-point in invariant mass distribution of the two leptons

$$
M_{\ell \ell}^{\text {cusp }}=m_{\delta}\left(1-\frac{m_{\psi}^{2}}{m_{\delta}^{2}}\right) e^{-\zeta}, M_{\ell \ell}^{\max }=m_{\delta}\left(1-\frac{m_{\psi}^{2}}{m_{\delta}^{2}}\right) e^{\zeta}, \cosh \zeta=\frac{M_{Z^{\prime}}}{2 m_{\delta}},
$$

where $\zeta$ is the rapidity of $\delta^{ \pm}$. Taking $M_{Z^{\prime}}=1 \mathrm{TeV}, m_{\delta}=450 \mathrm{GeV}$ and $m_{\psi}=50 \mathrm{GeV}$ as reference masses, we plot $M_{\ell \ell}$ in Fig. 9. One can read the two variables from this plot as $M_{\ell \ell}^{\text {cusp }}=280 \mathrm{GeV}$ and $M_{\ell \ell}^{\max }=708 \mathrm{GeV}$. Once we know the mass of gauge boson $Z^{\prime}$ from purely leptonic final states $p p \rightarrow Z^{\prime} \rightarrow \ell^{+} \ell^{-}$future [38], the masses of stable particle $\psi$ and its parent particle $\delta^{ \pm}$can be exactly solved from the two equations given above. The determined masses are $m_{\delta}=450.6 \mathrm{GeV}$ 

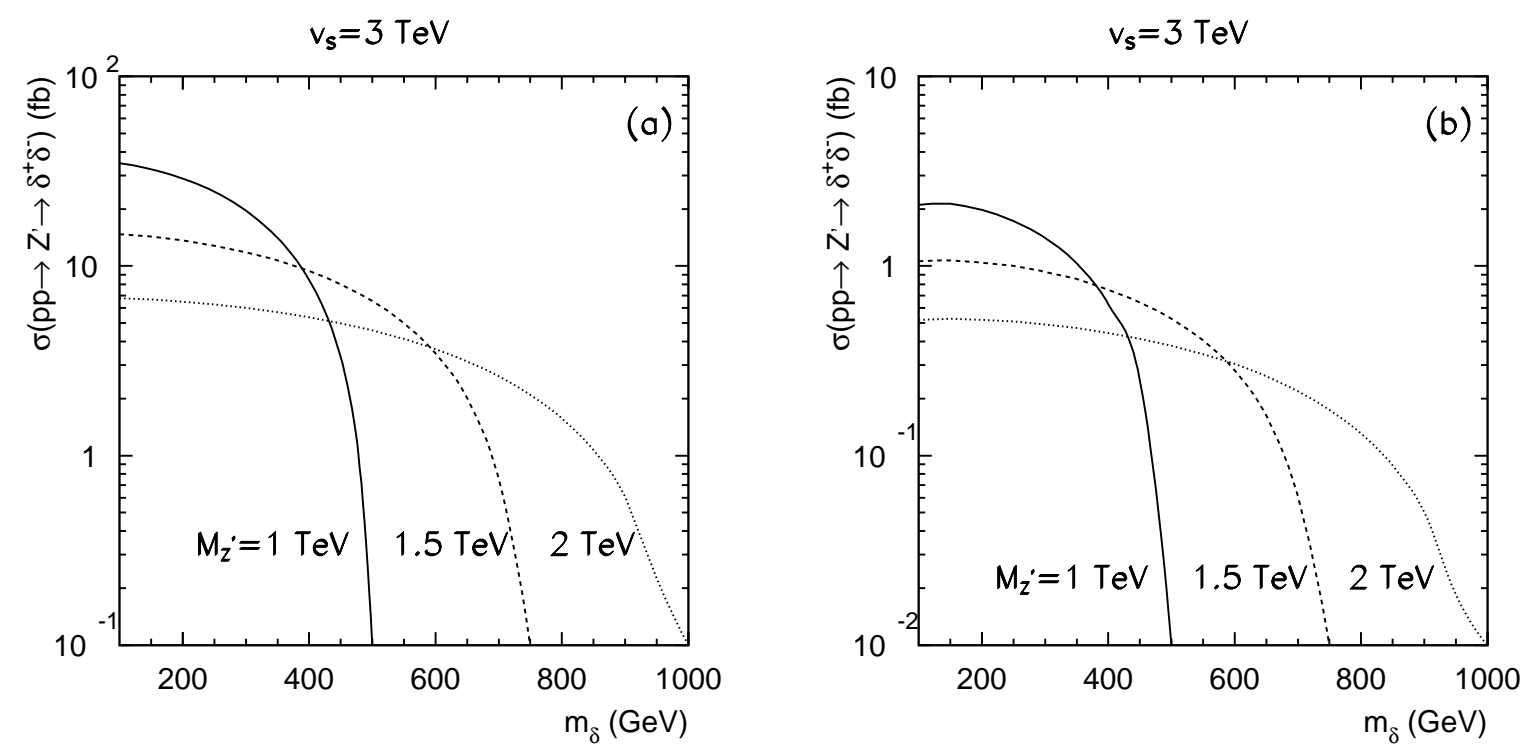

FIG. 8: Charged scalar $\delta^{ \pm}$pair production total cross section at the LHC versus its mass $m_{\delta}$ (a) without any cuts and (b) with all cuts and branching fraction of $\delta^{ \pm}$decay $B R\left(\delta^{ \pm} \rightarrow \ell^{ \pm} \psi\right)=30 \%$ taken from Fig. 6. The solid, dashed and dotted curves are for $M_{Z^{\prime}}=1,1.5,2 \mathrm{TeV}$ respectively, when $v_{\Phi}=3 \mathrm{TeV}$.

and $m_{\psi}=49.4 \mathrm{GeV}$ in this case.

\section{CONCLUSION AND SUMMARY}

In this paper we have established a hybrid seesaw mechanism to explain tiny neutrino masses and suggest a cold dark matter candidate. In this mechanism which is beyond the so-called Type I seesaw in the context of $B-L$ gauge symmetry extension of the Standard Model, the contributions of a new scalar doublet and two new fermion singlets appear at one-loop level. We have studied in detail the constraints on the model parameters from neutrino oscillation data, lepton flavor violating processes and cosmological observation. We have also explored the predictions on the decay branching ratios of the charged new scalar in each neutrino mass spectrum and showed the most optimistic scenarios where one could hope to distinguish the spectra using the properties of the decays. The typical signatures related to the new seesaw mechanism and dark matter candidate at the LHC are also studied. We summarize our main results in the following

- A radiative seesaw mechanism can be added to the minimal Type I seesaw with a local gauge symmetry $B-L$, a new scalar doublet and two new fermion singlets $\psi$ at loop level. 


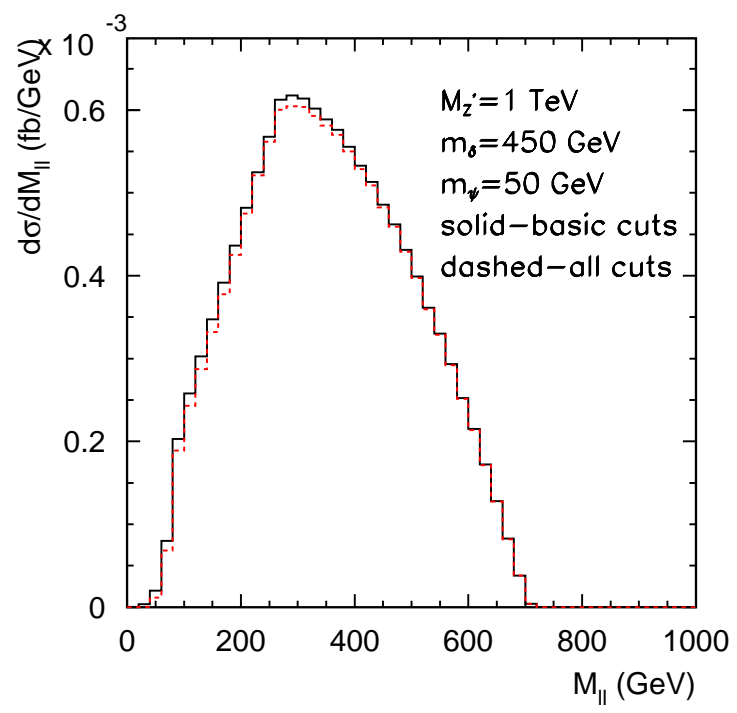

FIG. 9: Invariant mass distribution of two leptons for $p p \rightarrow Z^{\prime} \rightarrow \delta^{+} \delta^{-} \rightarrow \ell^{+} \ell^{-} \psi \psi$ production after basic cuts and all cuts, with $M_{Z^{\prime}}=1 \mathrm{TeV}, m_{\delta}=450 \mathrm{GeV}$ and $m_{\psi}=50 \mathrm{GeV}$.

- The decays of new scalar doublet are related to neutrino masses and mixings. We show the possibility to distinguish the neutrino spectra. The branching fractions can differ by one order of magnitude in $\mathrm{NH}$ case with $B R\left(\mu^{ \pm} \psi\right), B R\left(\tau^{ \pm} \psi\right) \gg B R\left(e^{ \pm} \psi\right)$, and a few times in the IH spectrum with $B R\left(e^{ \pm} \psi\right)>B R\left(\mu^{ \pm} \psi\right), B R\left(\tau^{ \pm} \psi\right)$ when the Majorana phase is ignored in $3+2$ mode.

- Considering effects of the Majorana phase, in NH the dominant channels swap from $\tau^{ \pm} \psi_{1}$ when $\chi \approx \pi / 2$ to $\mu^{ \pm} \psi_{1}$ when $\chi \approx 3 \pi / 2$ by a few times. In IH the dominant channels swap from $e^{ \pm} \psi_{1}$ when $\chi \approx \pi / 2$ to $\mu^{ \pm} \psi_{1}, \tau^{ \pm} \psi_{1}$ when $\chi \approx 3 \pi / 2$ by more than one order time of magnitude.

- The lightest new particle $\psi$ in the loop is stable and can be cold dark matter candidate because of $B-L$ gauge symmetry invariance. Cosmological observation constrains the masses of the new scalar and $\psi$ at a few hundred $\mathrm{GeV}$.

- Even when heavy Majorana neutrino pair production channel is not allowed from $Z^{\prime}$ gauge boson decay, in this framework, the new charged scalar can be pair produced at the LHC essentially.

- The masses of new scalar $\delta^{ \pm}$and missing particle $\psi$ can be well-determined in the production 


\begin{tabular}{|c|c|c|}
\hline Fields & Vertices & Couplings \\
\hline \multirow[t]{3}{*}{$\delta^{ \pm}$} & $Z_{\mu}^{\prime} \delta^{+}\left(p_{1}\right) \delta^{-}\left(p_{2}\right)$ & $-i g_{B L}\left(p_{1}-p_{2}\right)_{\mu}$ \\
\hline & $Z_{\mu} \delta^{+}\left(p_{1}\right) \delta^{-}\left(p_{2}\right)$ & $-i g_{2} \frac{\cos \left(2 \theta_{W}\right)}{2 \cos \left(\theta_{W}\right)}\left(p_{1}-p_{2}\right)_{\mu}$ \\
\hline & $A_{\mu} \delta^{+}\left(p_{1}\right) \delta^{-}\left(p_{2}\right)$ & $-i e\left(p_{1}-p_{2}\right)_{\mu}$ \\
\hline \multirow[t]{2}{*}{$\delta^{0}, F^{0}$} & $Z_{\mu}^{\prime} \delta^{0}\left(p_{1}\right) F^{0}\left(p_{2}\right)$ & $-g_{B L}\left(p_{1}-p_{2}\right)_{\mu}$ \\
\hline & $Z_{\mu} \delta^{0}\left(p_{1}\right) F^{0}\left(p_{2}\right)$ & $\frac{\sqrt{g_{1}^{2}+g_{2}^{2}}}{2}\left(p_{1}-p_{2}\right)_{\mu}$ \\
\hline \multirow[t]{9}{*}{$Z^{\prime}$} & $\bar{q}_{i} q_{i} Z^{\prime}$ & $-i Q_{B L}^{q} g_{B L} \gamma^{\mu}$ \\
\hline & $q_{1}=u, q_{2}=d$ & $Q_{B L}^{q}=\frac{1}{3}$ \\
\hline & $\bar{\ell} \ell Z^{\prime}$ & $-i Q_{B L}^{\ell} g_{B L} \gamma^{\mu}$ \\
\hline & $\ell=e, \mu, \tau$ & $Q_{B L}^{\ell}=-1$ \\
\hline & $\overline{N_{m}} N_{m} Z^{\prime}$ & $i g_{B L} \gamma^{\mu} \frac{\gamma_{5}}{2}$ \\
\hline & $N=\nu_{R}+\nu_{R}^{C}$ & \\
\hline & $\overline{\nu_{m}} \nu_{m} Z^{\prime}$ & $i g_{B L} \gamma^{\mu} \frac{-\gamma_{5}}{2}$ \\
\hline & $\nu=\nu_{L}+\nu_{L}^{C}$ & \\
\hline & $\phi^{0}\left(p_{1}\right) \phi^{0}\left(p_{2}\right) Z^{\prime}$ & $-i 2 g_{B L}\left(p_{1}-p_{2}\right)_{\mu}$ \\
\hline \multirow[t]{4}{*}{$\psi$} & $\bar{\nu} \delta^{0} \psi$ & $-i Y_{\psi} \frac{1}{\sqrt{2}} P_{R}$ \\
\hline & $\psi=\psi_{R}+\psi_{R}^{C}$ & \\
\hline & $\bar{\nu} F^{0} \psi$ & $-Y_{\psi} \frac{1}{\sqrt{2}} P_{R}$ \\
\hline & $\bar{\ell} \delta^{-} \psi$ & $i Y_{\psi} P_{R}$ \\
\hline
\end{tabular}

TABLE II: Feynman rules. The momenta are all assumed to be incoming.

topology $p p \rightarrow Z^{\prime} \rightarrow \delta^{+} \delta^{-} \rightarrow \ell^{+} \ell^{-} \psi \psi$ in terms of properties of visible SM charged leptons invariant mass distribution.

\section{Acknowledgment}

We acknowledge T. Han for providing his Fortran codes HANLIB for our calculations. T. L. would like to thank X. G. He for helpful discussions. This work is supported in part by the DOE under grant No. DE-FG02-91ER40626 (T.L.).

\section{Appendix A: Feynman Rules}

We summarize the Feynman rules for our model in Table. II. 
[1] Q. P. Ahamed et al. [SNO Collaboration], Phys. Rev. Lett. 89, 011301 (2002).

[2] For a review, see: C. K. Jung et al., Ann. Rev. Nucl. Part. Sci. 51, 451 (2001).

[3] K. Eguchi et al. [KamLAND Collaboration], Phys. Rev. Lett. 90, 021802 (2003).

[4] M. H. Ahn et al. [K2K Collaboration], Phys. Rev. Lett. 90, 041801 (2003).

[5] E. Komatsu et al., arXiv: 1001.4538 [astro-ph.CO].

[6] J. Angle et al. [XENON Collaboration], Phys. Rev. Lett. 100, 021303 (2008).

[7] Z. Ahmed et al. [CDMS Collaboration], arXiv: 0802.3530 [astro-ph].

[8] R. Bernabei et al. [DAMA Collaboration], Eur. Phys. J. C56, 333 (2008).

[9] Z. Ahmed et al. [The CDMS-II Collaboration and CDMS-II Collaboration], Science 327 (2010) 16191621.

[10] C. E. Aalseth et al. [CoGeNT Collaboration], arXiv:1002.4703 [astro-ph.CO].

[11] P. Minkowski, Phys. Lett. B67, 421 (1977); T. Yanagida, in Workshop on Unified Theories, KEK report 79-18 p.95 (1979); M. Gell-Mann, P. Ramond, R. Slansky, in Supergravity (North Holland, Amsterdam, 1979) eds. P. van Nieuwenhuizen, D. Freedman, p.315; S. L. Glashow, in 1979 Cargese Summer Institute on Quarks and Leptons (Plenum Press, New York, 1980) eds. M. Levy, J.-L. Basdevant, D. Speiser, J. Weyers, R. Gastmans and M. Jacobs, p.687; R. Barbieri, D. V. Nanopoulos, G. Morchio and F. Strocchi, Phys. Lett. B90, 91 (1980); R. N. Mohapatra and G. Senjanovic, Phys. Rev. Lett. 44, 912 (1980); G. Lazarides, Q. Shafi and C. Wetterich, Nucl. Phys. B181, 287 (1981).

[12] W. Konetschny and W. Kummer, Phys. Lett. B70, 433 (1977); T. P. Cheng and L. F. Li, Phys. Rev. D22, 2860 (1980); G. Lazarides, Q. Shafi and C. Wetterich, Nucl. Phys. B181, 287 (1981); J. Schechter and J. W. F. Valle, Phys. Rev. D22, 2227 (1980); R. N. Mohapatra and G. Senjanovic, Phys. Rev. D23, 165 (1981).

[13] R. Foot, H. Lew, X. G. He and G. C. Joshi, Z. Phys. C44, 441 (1989).

[14] P. Fileviez Pérez, T. Han, T. Li, Phys. Rev. D80, 073015 (2009).

[15] A. Zee, Phys. Lett. B93, 389 (1980), Erratum-ibid. B95, 461(1980).

[16] A. Zee, Phys. Lett. B161, 141 (1985); A. Zee, Nucl. Phys. B264, 99 (1986); K. S. Babu, Phys. Lett. B203, 132 (1988).

[17] E. Ma and U. Sarkar, Phys. Rev. Lett. 80, 5716 (1998).

[18] L. M. Krauss, S. Nasri and M. Trodden, Phys. Rev. D67, 085002 (2003); K. Cheung and O. Seto, Phys. Rev. D69, 113009 (2004); M. Aoki, S. Kanemura and O. Seto, Phys. Rev. Lett. 102, 051805 (2009); M. Aoki, S. Kanemura and O. Seto, Phys. Rev. D80, 033007 (2009).

[19] P. Fileviez Pérez, M. B. Wise, Phys. Rev. D80, 053006 (2009).

[20] L. L. Honorez, E. Nezri, J. F. Oliver, M. H. G. Tytgat, JCAP 0702, 028 (2007); D. M. Dolle and S. Su, Phys. Rev. D80, 055012 (2009).

[21] E. Ma, Phys. Rev. D73, 077301 (2006). 
[22] J. Kubo, E. Ma and D. Suematsu, Phys. Lett. B642, 18 (2006); J. Kubo and D. Suematsu, Phys. Lett. B643, 336 (2006).

[23] D. Suematsu, T. Toma, T. Yoshida, Phys. Rev. D79, 093004 (2009).

[24] B. Pontecorvo, Zh. Eksp. Teor. Fiz. (JETP) 33, 549 (1957); ibid. 34, 247 (1958); ibid. 53, 1717 (1967); Z. Maki, M. Nakagawa, S. Sakata, Prog. Theor. Phys. 28, 870 (1962).

[25] T. Schwetz, M. Tortola and J. W. F. Valle, New J. Phys. 10, 11301 (2008).

[26] J. A. Casas and A. Ibarra, Nucl. Phys. B618, 171 (2001).

[27] X. G. He, T. Li and W. Liao, Phys. Rev. D81, 033006 (2010).

[28] L. Alvarez-Gaume et al., Phys. Lett. B667, 1 (2008).

[29] G. Bertone, D. Hooper and J. Silk, Phys. Rep. 405, 279 (2005).

[30] M. S. Carena, A. Daleo, B. A. Dobrescu and T. M. P. Tait, Phys. Rev. D70, 093009 (2004); G. Cacciapaglia, C. Csaki, G. Marandella and A. Strumia, Phys. Rev. D74, 033011 (2006).

[31] P. Langacker, arXiv:0801.1345 [hep-ph].

[32] M. S. Carena, A. Daleo, B. A. Dobrescu, T. M. P. Tait, Phys. Rev. D70, 093009 (2004); F. Petriello and S. Quackenbush, Phys. Rev. D77, 115004 (2008).

[33] See for example: K. Huitu, S. Khalil, H. Okada and S. K. Rai, Phys. Rev. Lett. 101, 181802 (2008); J. A. Aguilar-Saavedra, arXiv:0905.2221 [hep-ph]; L. Basso, A. Belyaev, S. Moretti and C. H. ShepherdThemistocleous, arXiv:0812.4313 [hep-ph]; N. Okada, O. Seto, arXiv:1002.2525 [hep-ph].

[34] CTEQ Collaboration, J. Pumplin, D. R. Stump, J. Huston, H. L. Lai, P. M. Nadolsky and W. K. Tung, JHEP 0207, 012 (2002).

[35] J. Alwall, P. Demin, S. d. Visscher, R. Frederix, M. Herquet, F. Maltoni, T. Plehn, D. L. Rainwater, T. Stelzer, JHEP 0709, 028 (2007).

[36] CMS TDR: CMS Physics: Technical Design Report V.2: Physics Performance, CERN-LHCC-2006-021.

[37] T. Han, I. W. Kim, J. Song, arXiv: 0906.5009 [hep-ph].

[38] See for example: L. Basso, A. Belyaev, S. Moretti, G. M. Pruna and C. H. Shepherd-Themistocleous, arXiv:1002.3586 [hep-ph]. 